International Journal of Engineering \& Technology, $7(2.25)(2018) 95-99$
SPC
International Journal of Engineering \& Technology
Website: $w w w . s c i e n c e p u b c o . c o m / i n d e x . p h p / I J E T$
Research paper

\title{
Edge detection for detection of brain tumour in CT images
}

\author{
T. R. Thamizhvani ${ }^{1}{ }^{*}$, A. Josephin Arockia Dhivya ${ }^{1}$, S. Akshaya ${ }^{1}$, K. Dhanalakshmi ${ }^{1}$, R. Chandrasekaran ${ }^{1}$, \\ Josline Elsa Joseph ${ }^{1}$ \\ ${ }^{1}$ Department of Biomedical Engineering, Vels University, Pallavaram, Chennai-600117, India \\ *Corresponding author E-mail: thamizhvani.se@velsuniv.ac.in
}

\begin{abstract}
Brain tumour can be defined as the continuous and uncontrolled growth of the cells in the regions of brain. Analysis and detection of brain tumours from the computed tomography images can be performed by various image processing algorithms. Edge detection is special type of image processing technique, which uses operators for functioning. The Computed Tomography images are obtained from the standard data-base which undergoes pre-processing technique. Contrast adjustment is performed to enhance the region of brain tumour. Edge operators of different types are applied to the images for identification of the boundary of the brain tumour region. Appropriate edge operator for de-termination of the boundary is defined by comparing the image quality and accuracy parameters. These parameters illustrate that canny oper-ator is described to be more definite for the detection and analysis of the boundary and region of brain tumour in Computed Tomography images.
\end{abstract}

Keywords: Edge Operators; Computed Tomography (CT); Peak Signal to Noise Ratio (PSNR); F-Measure.

\section{Introduction}

Imaging technology in Medicine helps the doctors to diagnose and detect the interior portions of the body easily. Imaging acts as a keyhole for surgeries in reaching the interior parts. CT scanner, Ultrasound and Magnetic Resonance Imaging replace $\mathrm{x}$-ray imaging by making the doctors to visualise the body's elusive third dimension. Different image processing techniques are used for analysis of internal structures. There are various advantages of digital imaging techniques such as information is not changed when it is reproduced again and retains the originality and enhancement of images makes diagnosis easier for physicians and also to obtain quick comparison of images [1].

Edge detection is a special type of technique used for defining the edges and boundaries of the anatomical structures of the human body. Edge detection includes a variety of mathematical approaches that aims at identifying points in a digital image. The points at which image brightness changes sharply are typically organized into a set of curved line segments said to be edges [4]. Edge detection is a fundamental tool in image processing, machine vision and computer vision, especially in the areas of feature detection and feature extraction. Edge detection can also be used for detection of tumour edges or discontinuities, which is helpful during surgeries [9].

Brain tumour is an abnormal growth of cells within the brain, which can be cancerous or non-cancerous (benign). It is generally caused by abnormal and uncontrolled cell division primarily occurs either in the brain (neurons, glial cells (astrocytes, oligodendrocytes, ependymal cells), lymphatic blood vessels), cranial nerves (myelinproducing Schwann cells), in brain envelopes (meninges), skull, pituitary and pineal gland or may be spread from cancers primarily located in other organs (metastatic tumours).

Tumours can be classified into benign and malignant based on the structural appearance and functioning. Benign brain tumours usually have an obvious border or edge and don't spread to other parts of the body. However, benign tumours can supress sensitive areas of the brain and cause serious health problems. Malignant brain tumours are defined to be a serious threat to life. They are likely to grow rapidly and crowd or invade the nearby healthy brain tissue. Cancer cells may break away from malignant brain tumours and spread to other parts of the brain or to the spinal cord. Symptoms of brain tumour include headache, muscular weakness, dizziness, reduced sensation to touch and abnormal cognitive functioning. Brain tumour can be treated either through surgery, radiation therapy or chemotherapy which is based on the type, grade and size of the tumour [7].

With a surge in the brain related ailments in the world, every year over 2,500 of the Indian children suffer from medulloblastoma, a paediatric malignant primary brain tumour which spreads through the cerebrospinal fluid (CSF) and frequently metastasize to different locations along the surface of the brain and spinal cord. According to World Health Organisation (WHO) in India, every year 40,000-50,000 persons are diagnosed with brain tumour. Of these 20 percent are children. The physicians say that if the cases are detected early, then 90 percent of the medulloblastoma cases are curable, provided the treatment protocol is followed correctly.

In this paper, CT images of brain tumour are derived from the authorised database for which different edge detection techniques are applied and compared. Based on this comparison, effective method in edge detection process is identified for the detection of the boundaries of brain tumour. This process is defined for the easy diagnosis and detection of brain tumour structures.

\section{Literature review}

Mamta Juneja (2009) defines the comparison of various edge detection techniques using normal images. Performance evaluation of edge detection operators is obtained based on the statistical features. Relative frequency of the first order and second order edge opera- 
tors are calculated after removing the noise. The statistical parameters are calibrated using the relative frequencies of the different operators.

Shrivakshan.G.T (2012) describes the shark fish classification with the help of various edge detection techniques. The edge detection operators considered for comparison and classification are Roberts, Prewitt, Sobel, Laplacian and Laplacian of Gaussian. The advantages and disadvantages of the operators are defined for analysis and classification. Operators are compared in this paper based on the statistical features such as mean and standard deviation [5]. Ed-Edily Mohd Azhar (2014) defines the algorithm for localisation and segmentation of brain tumours from Magnetic Resonance Imaging (MRI) images. MRI images are subjected to pre-processing and edge detection for isolation of the brain tumour. Modified Histogram clustering is performed in the paper for accurate localisation of the brain tumour. Comparison features such as pixel area and mean of HSV plane are determined for the images to identify the brain tumours structures. Optimization of the brain tumours in more than 100 neuroimages is carried out with this algorithm. In this paper, edge detection operators are used for the analysis and detection of brain tumour from Computed tomography images [6].

\section{Methodology}

In this methodology, detection of boundaries of brain tumour in Computed tomographic (CT) images is performed using edge detection techniques. Computed Tomographic (CT) images of the brain tumour are obtained from the standard database for analysis. Pre-processing of the database images are carried out to enhance the regions of brain tumour. Different edge detection operators are applied for the brain tumour images. The suitable edge operator for brain tumour detection is analysed by comparing the parameters such as mean square error, root mean square, peak signal to noise ratio and F-measure. This algorithm is mainly for early diagnosis and detection of definite boundaries of brain tumours using edge detection techniques.

\subsection{Contrast adjustment}

Contrast can be defined as difference in brightness of the regions in an image. Contrast adjustment is mainly used to enhance the regions of interest for further analysis. Contrast adjustment is used to remap the intensity values for the complete display range in the images. Images with high and good contrast possess accurate differences between all ranges of colour. In this algorithm, brain tumour region in the CT image is enhanced with the help of contrast adjustment.

\subsection{Edge operators}

Edge detection is a special type of technique used for defining the edges and boundaries of the anatomical structures of the human body. Edge detection includes a variety of mathematical approaches that aims at identifying points in a digital image. In Edge detection process, operators are used in defining the edges or boundaries of the specified region of interest. Four types of edge operators are used for detection of brain tumours. They are sobel, prewitt, canny and Roberts operator.

\subsubsection{Sobel operator}

Sobel operator can be defined as sobel filter which is used in image processing particularly for determination of edges of a region. Technically, sobel operator is a discrete differentiation operator providing an approximation of the gradient of the image intensity function. Sobel operator is described below where * denotes the two dimensional signal processing convolution operation [2].
$G_{x}=\left[\begin{array}{ccc}+1 & 0 & -1 \\ +2 & 0 & -2 \\ +1 & 0 & -1\end{array}\right] * \mathrm{~A}$ and $G_{y}\left[\begin{array}{ccc}+1 & +2 & +1 \\ 0 & 0 & 0 \\ -1 & -2 & -1\end{array}\right] * \mathrm{~A}$

\subsubsection{Prewitt operator}

Prewitt operator is used in the image processing particularly within edge detection algorithms. This operator is based on convolving the image with a small, separable and integer valued filtered in horizontal and vertical direction. Therefore relatively used in expensive in terms of computation like sobel operators. Prewitt operator is defined below where $*$ denotes one dimensional convolution operation.

$G_{x}=\left[\begin{array}{ccc}-1 & 0 & +1 \\ -1 & 0 & +1 \\ -1 & 0 & +1\end{array}\right] * A$ and $G_{y}=\left[\begin{array}{ccc}-1 & -1 & -1 \\ 0 & 0 & 0 \\ +1 & +1 & +1\end{array}\right] * A$

\subsubsection{Robert's operator}

Roberts operator is used in image processing and computer vision for edge detection. This Operator can be defined as one of the first edge detector. Roberts operator is mainly used to approximate the gradient of an image through discrete differentiation which is achieved by computing the sum of the squares of the difference between diagonally adjacent pixels [3].

$\mathrm{Gx}=\left[\begin{array}{cc}+1 & 0 \\ 0 & -1\end{array}\right] \mathrm{Gy}=\left[\begin{array}{cc}0 & +1 \\ -1 & 0\end{array}\right]$

\subsubsection{Canny operator}

Canny operator is used in the multi stage algorithm to detect a wide range of edges in images. Canny algorithm uses four filters to detect horizontal, vertical and diagonal edges in the blurred image. Canny operator is shown below where $*$ represents one dimensional convolution operation [10].

$\mathrm{B}=\frac{1}{159}\left[\begin{array}{ccccc}2 & 4 & 5 & 4 & 2 \\ 4 & 9 & 12 & 9 & 4 \\ 5 & 12 & 15 & 12 & 5 \\ 4 & 9 & 12 & 9 & 4 \\ 2 & 4 & 5 & 4 & 2\end{array}\right] * \mathrm{~A}$

\subsection{Image quality and accuracy parameters}

Image quality and accuracy parameters such as mean squared error, root mean square, Peak signal to noise ratio and $\mathrm{F}$-measure are determined for analysis and identification of the accurate edge detection operator for detection of brain tumours in CT images [2,11].

\subsubsection{Mean squared error}

Mean squared error (MSE) or mean squared deviation (MSD) of an estimator measures the average of the squares of the errors or deviations that is the difference between the estimators. MSE is a measure of the quality of an estimator which is always non-negative and it is better when the values are closer to zero. In this, equation (1) describes the mean squared error.

$\operatorname{MSE}=\frac{1}{n} \sum_{i=1}^{n}\left(y_{i-} \tilde{y}_{i}\right)^{2}$

\subsubsection{Root mean square}

Root Mean Square is defined as the square root of mean square. The RMS is also known as the quadratic mean and is a practice case of the generalized mean with the exponent. RMS can also be defined for continuously varying function in terms of an integral of the 
squares of the instantaneous value during a cycle. Root mean square is defined in the equation (2)

$$
\mathrm{RMS}=\sqrt{\frac{x_{1}^{2}+x_{2}{ }^{2}+\cdots+x_{N}{ }^{2}}{N}}
$$

\subsubsection{PSNR (peak signal to noise ratio)}

PSNR is an engineering term for the ratio between the maximum possible power of a signal and the power of corruption noise that affects the fidelity of its representation. Because many signals have a very wide dynamic range, PSNR is usually expressed in terms of the logarithmic decibel scale. PNRS is most commonly used to measure the quality of reconstruction of lossy compression codecs. (Example: Image compression). Peak signal to noise ratio is described in the equation (3)

$$
\mathrm{PSNR}=10 \times \log \left(\frac{255^{2}}{M S E}\right)
$$

\subsubsection{F-measure}

F-measure defines the accuracy of the test performed. This measure considers precision and recall as the primary factor. These two factors compute the accurate nature of the test. F-measure of all the four edge detectors is determined using the precision and recall sample values. Accurate edge detection method can be defined with the help of this measure [12]. F- measure is described below in the equation (4)

$$
F-\text { measure }=2 \cdot \frac{\text { Precision } . \text { Recall }}{\text { Precision }+ \text { Recall }}
$$

Detection of brain tumour from the $\mathrm{CT}$ images using edge detection algorithm. Various edge operators are used from which the absolute operator for brain tumour detection is obtained by comparing the image quality parameters.

\section{Results and discussion}

In this paper, methodology depends on the identification of accurate edge operator for the detection of brain tumours in CT images. Computed tomographic images from an authorised database are considered for analysis. CT brain tumour images undergo pre-processing technique, contrast adjustment to enhance the brain tumour region. After pre-processing, edge operators are applied to the images for segregation of brain tumour regions in the image. Original and pre-processed Brain tumour images are shown in the figure 1(a) and $1(\mathrm{~b})$ respectively.

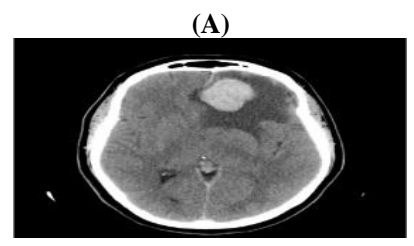

(B)

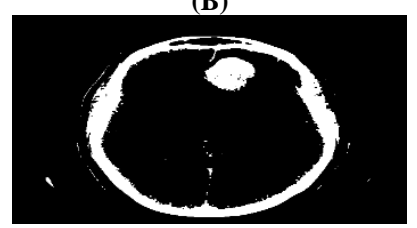

Fig. 1: A) Brain Tumour CT Image. B) Pre-Processed Image.

Edge operators such as sobel, prewitt, canny and Roberts are applied for the pre-processed images. These four different operators are used for detection of boundaries of the brain tumour in CT images. Each edge operator produces a specific result with variations in the discontinuties of the pixels in boundaries. These variations can be compared with the image quality parameters from which the accurate edge operator for brain tumour detection can be determined. The resultant images of four different operators applied after pre-prcoessing for identifciation of brain tumour is shown in the figure 2 .
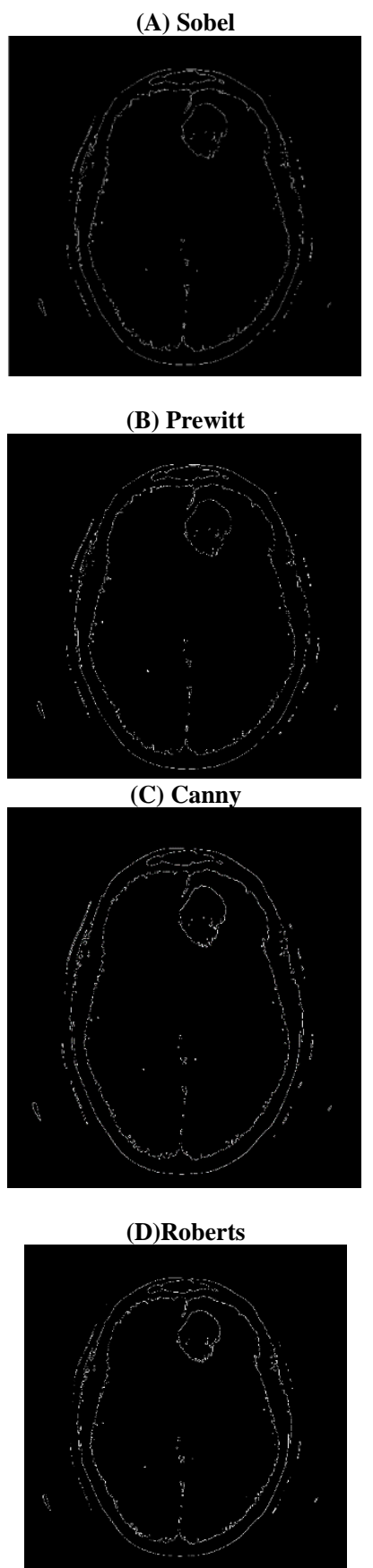

Fig. 2: Edge Operator Results for Brain Tumour CT Images.

Comparison of edge detection operators are carried out with the help of certain image quality parameters such as mean squared error, root mean square, Peak signal to noise ratio and F-measure. In this all the parameters are compared based on which canny operator is defined to be more accurate for the detection and identification of the brain tumour regions in the CT image. Image quality and accuracy parameters of the four operators are compared and illustrated graphically in the figure $3,4,5$ and 6 respectively. 


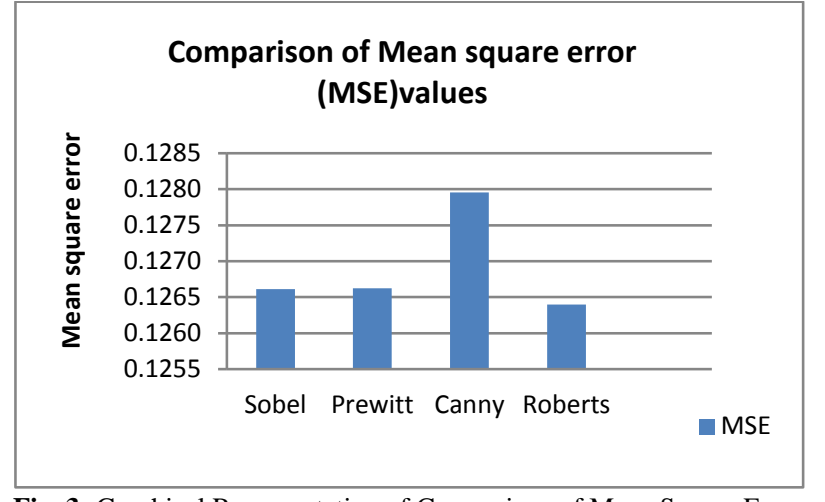

Fig. 3: Graphical Representation of Comparison of Mean Square Error

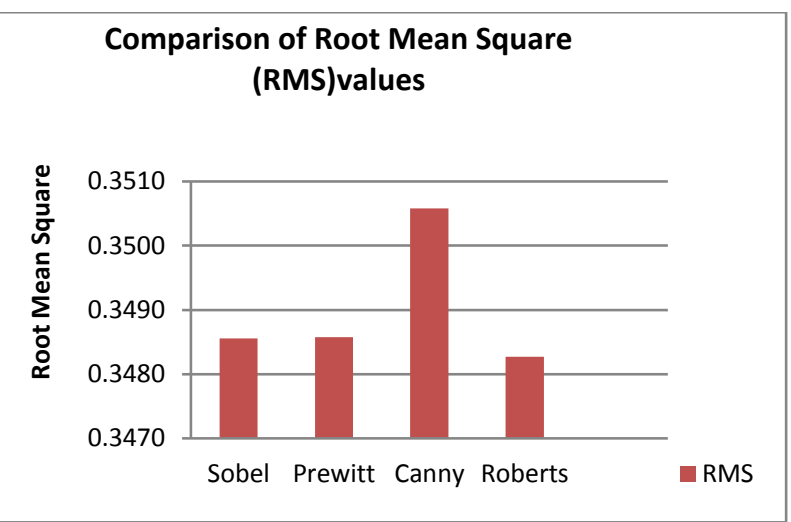

Fig. 4: Graphical Representation of Comparison of Root Mean Square.

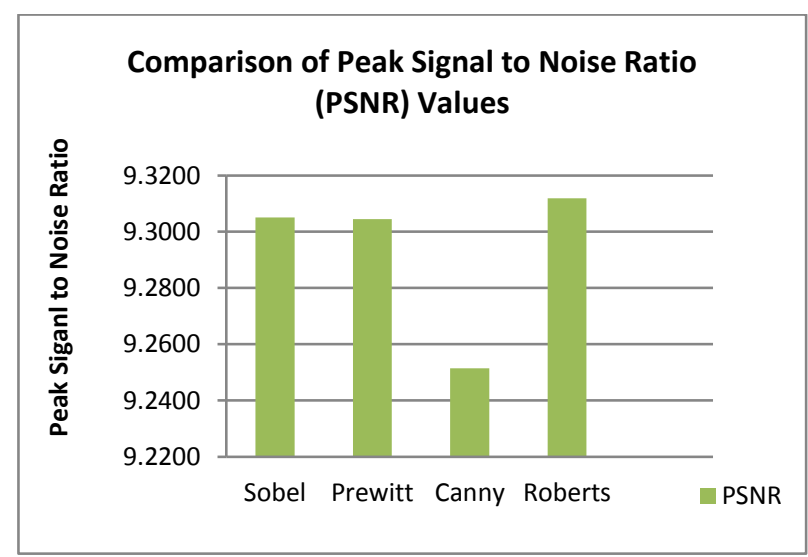

Fig. 5: Graphical Representation of Comparison of Peak Signal to Noise Ratio.

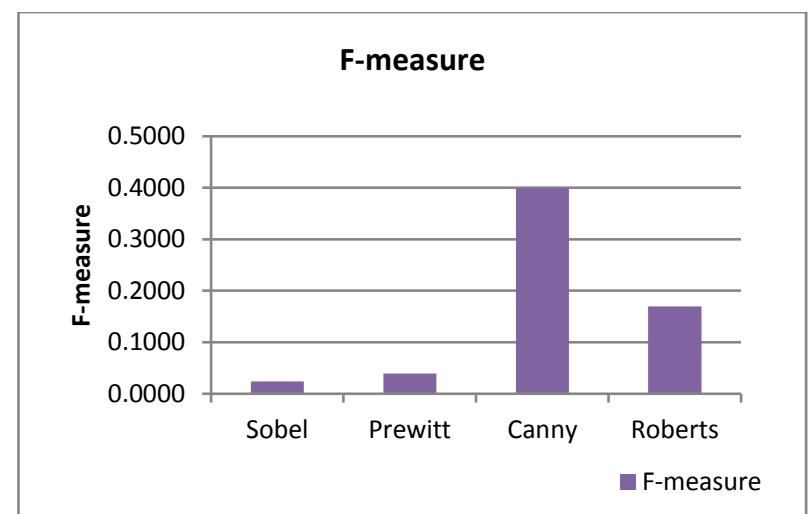

Fig. 6: Graphical Representation of Comparison of F-Measure Values.

Edge detection algorithm with different operators is applied to brain tumour CT images for determination of the region of brain tumour. For identification of accurate operator for detection of the brain tumour region is carried out by comparing various quality parameters.

\section{Conclusions}

Brain tumour is a type of uncontrolled growth of cells in the brain regions which can be diagnosed using different imaging modalities especially computed tomographic (CT) images. Analysis of brain tumours in the CT images can be obtained by various image processing techniques. One among them is edge detection process which is simple and effectively carried out with the help of operators. Four different operators are applied to the CT images after preprocessing. In pre-processing, contrast adjustment is performed to enhance the images specially the region of brain tumour. With the process of edge operators, the boundary of the brain tumour region is detected. The operator effective for the detection of the boundary is identified by comparison of the image quality parameters such as mean square error, root mean square, peak signal to noise ratio and F-measure. These parameters are graphically represented which defines canny operator to be more appropriate for defining the boundary of the brain tumour region from the computed tomographic images. Thus canny operator can be used for analysis of brain tumours in computed tomography (CT) images.

\section{References}

[1] Mohammed Roushdy; "Comparative study of edge detection algorithms applying on the Grayscale Noisy Image using Morphological Filter", GVIP Journal, Vol. 6, No. 4, (2006), pp. 17-23, available online:https://pdfs.seman-

ticscholar.org/5150/91c8f11926ee19dbb6ea0c3f8cfe7fb5b10e.pdf.

[2] Samir kumar Bandyopadhyay; "Edge detection from CT images of lung", International Journal of Engineering Science \& Advanced Technology, Vol. 2, No. 1,(2012), pp. 34-37, available online: https://pdfs.seman-

ticscholar.org/5c1a/4894989e6c228dee5b5fce63cfecf892fa70.pdf

[3] Li Bin,Mehdi Samiei Yeganeh; "Comparision for image edge detection algorithms", IOSR Journal of Computer Engineering, Vol. 2 No. 6, (2012), pp. 1-4, available online: www.iosrjournals.org/iosrjce/papers/vol2-issue6/A0260104.pdf

[4] Anurag Sharma, Pankaj Sharma, Rashmi, Hardeep Kumar; "Edge detection of medical images using morphological algorithms", International Journal of Engineering Sciences \& Emerging Technologies, Vol. 2, No. 2, (2012), pp.66-72, available online: www.ijeset.com/media/0001/9I4-IJESET-EDGE-DETECTION.pdf

[5] G.T.Shrivakshan; "A comparision of various egde detection techniques used in image processing", International Journal of Computer Sciences, Vol. 9, No. 5, (2012), pp. 269-276, available online: https://www.ijcsi.org/papers/IJCSI-9-5-1-269-276.pdf.

[6] Ed-Edily Mohd Azhari, Muhd Mudzakkir Mohd.Hatta, Zaw zaw Htike and shoon lei win; "Brain tumor detection and localization in magnetic resonance imaging", International Journal of Information Technology Convergence and Services, Vol. 4, No. 1,(2014), pp. 111, available online : https://pdfs.semanticscholar.org/bfd3/87d75de4e9c610799a848eace7fd3c278522.pdf.

[7] HowardLee, Yi-Ping PhoebeChen, "Image based computer aided diagnosis system for cancer detection", Expert Systems with Applications, Vol. 42, No. 12, (2015), pp.5356-5365, available online: https://www.sciencedirect.com/science/article/pii/S0957417415000986.

[8] Ling Zhang, Vissagan Gopalakrishnan, Le Lu, Ronald M. Summers, Joel Moss, Jianhua Yao, "Self-Learning to Detect and Segment Cysts in Lung CT Images without ManualAnnotation", Computer Vision and Pattern Recognition, Vol. 3, (2018), pp. 1-4, available online: https://arxiv.org/abs/1801.08486.

[9] Abdulrahman Moffaq Alawad, Farah Diyana Abdul Rahman, Othman O. Khalifa, Norun Abdul Malek, "Fuzzy Logic Based Edge Detection Method for Image Processing", International Journal of Electrical and Computer Engineering, Vol. 8, No.3, (2018), pp. 2-5, available online: http: // www.iaescore.com /journals /index.php /IJECE /article/view/11794.

[10] Weibin Rong, Zhanjing Li, Wei Zhang, Lining Sun, “An improved Canny edge detection algorithm", Proceedings of the International Conference on Mechatronics and Automation, (2014), pp. 577-582, http:// dx.doi.org/ 10.1109/ICMA.2014.6885761.

[11] Haijiang Hu, Juju Hu, Qing Tao, Shuqin Liu, "Simple edge detection method for vascular tree angiogram", Proceedings of the Advanced 
Information Technology, Electronic and Automation Control Conference (IAEAC), IEEE, (2015), pp. 433-436, http:// dx.doi.org/ 10.1109/IAEAC.2015.7428590.

[12] Radhakrishna Achanta, Sheila Hemami, Francisco Estrada, Sabine Susstrunk, "Frequency-tuned salient region detection", Proceedings of the Computer Vision and Pattern Recognition conference (CVPR), IEEE,(2009), pp.1597 - 1604, http:// dx.doi.org/ 10.1109/CVPR.2009.5206596. 